\section{Contributing Authors}

\section{Franklin Allen}

Nippon Life Professor of Finance and Economics University of Pennsylvania 2253 Steinberg Hall-Dietrich Hall 3620 Locust Walk, Suite 2300 Philadelphia, PA 19104-6367

allenf@wharton.upenn.edu

\section{Geert Bekaert}

L. Cooperman Professor of Finance and Economics

Columbia Business School

Uris Hall 802

3022 Broadway

New York, NY 10027

gb241@columbia.edu

\section{Valerie R. Bencivenga}

University of Texas at Austin

1 University Station C3100

Austin, TX 78712

benciven@eco.utexas.edu

\section{Nicola Cetorelli}

Research Department

Federal Reserve Bank of Chicago

230 South LaSalle Street

Chicago, IL 60604-1413

ncetorelli@frbchi.org

\section{Campbell R. Harvey}

Fuqua School of Business

Duke University

Durham, NC 27708-0120

cam.harvey@duke.edu

\section{Peter Blair Henry}

Graduate School of Business

Stanford University

Stanford, CA 94305-5015

pbhenry@Stanford.edu

\section{Ross Levine}

Curtis L. Carlson Chair in Finance

Carlson School of Management

University of Minnesota

CSOM: 3-257

321 19th Avenue South

Minneapolis, MN 55455

RLevine@csom.umn.edu

\section{Christian T. Lundblad}

Finance Department

Kelley School of Business

Indiana University

1309 East Tenth Street

Bloomington, IN 47405-1701

clundbla@indiana.edu

\section{Raghuram G. Rajan}

Joseph L. Gidwitz Professor of Finance

Graduate School of Business

University of Chicago

1101 East 58th Street

Chicago, IL 60637

rajan@gsb.uchicago.edu

Peter L. Rousseau

Economics Department

Vanderbilt University

Box 1819 Station B

Nashville, TN 37235

peter.1.rousseau@vanderbilt.edu

Philip E. Strahan

Finance Department

Boston College

324b Fulton Hall

140 Commonwealth Avenue

Chestnut Hill, MA 02467

philip.strahan@bc.edu

David C. Wheelock

Research Department

Federal Reserve Bank of St. Louis

411 Locust Street

St. Louis, MO 63102

David.C.Wheelock@stls.frb.org

\section{Eugene N. White}

Economics Department

Rutgers University

New Brunswick, NJ 08901-1248

white@economics.rutgers.edu

\section{Luigi Zingales}

Robert C. McCormack Professor of

Entrepreneurship and Finance

Graduate School of Business

University of Chicago

1101 East 58th Street

Chicago, IL 60637

luigi.zingales@gsb.uchicago.edu 
REVIEW

2 July/August 2003 